\title{
Probing the complex environments of GRB host galaxies and intervening systems: high resolution spectroscopy of GRB050922C*
}

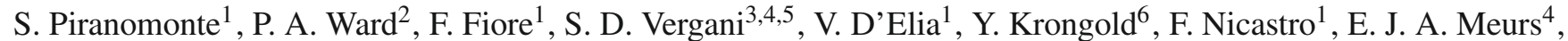 \\ G. Chincarini ${ }^{5,7}$, S. Covino ${ }^{5}$, M. Della Valle ${ }^{8,9,10}$, D. Fugazza ${ }^{5}$, L. Norci ${ }^{3}$, L. Sbordone ${ }^{11}$, L. Stella ${ }^{1}$, G. Tagliaferri ${ }^{5}$, \\ D. N. Burrows ${ }^{12}$, N. Gehrels ${ }^{13}$, P. Goldoni ${ }^{14,15}$, D. Malesani ${ }^{16}$, I. F. Mirabel ${ }^{17, \star \star}$, L. J. Pellizza ${ }^{18}$, and R. Perna ${ }^{19}$
}

1 INAF-Osservatorio Astronomico di Roma, via Frascati 33, 00040 Monte Porzio Catone (RM), Italy e-mail: piranomonte@oa-roma.inaf.it

2 Mullard Space Science Laboratory, University College London, Holmbury St. Mary, Dorking Surrey, RH56NT, UK

3 Dublin Institute for Advanced Studies, 31 Fitzwilliam Place, Dublin 2, Ireland

4 School of Physical Sciences and NCPST, Dublin City University, Glasnevin, Dublin 9, Ireland

5 INAF-Osservatorio Astronomico di Brera, via E. Bianchi 46, 23807 Merate (LC), Italy

6 Instituto de Astronomia, Universidad nacional Autonoma de Mexico, Apartado Postal 70-264, 04510 Mexico DF, Mexico

7 Università degli Studi di Milano Bicocca, Piazza della Scienza 3, 20126 Milano, Italy

8 INAF-Osservatorio Astronomico di Capodimonte, Salita Moiariello 16, 80131 Napoli, Italy

9 International Center for Relativistic Astrophysics Network, 65122, Pescara, Italy

${ }_{10}$ European Southern Observatory, Karl-Schwarzschild-Strasse 2, 85748 Garching bei München, Germany

11 GEPI, Observatoire de Paris, CNRS, Université Paris Diderot, Place Jules Janssen, 92190 Meudon, France

12 Department of Astronomy and Astrophysics, Pennsylvania State University, University Park, Pennsylvania 16802, USA

13 NASA-Goddard Space Flight Center, Greenbelt, Maryland, 20771, USA

14 APC, Laboratoire Astroparticule et Cosmologie, UMR 7164, 11 place Marcelin Berthelot, 75231 Paris Cedex 05, France

15 CEA Saclay, DSM/DAPNIA/Service d'Astrophysique, 91191 Gif-sur-Yvette, France

${ }_{17}$ Dark Cosmology Centre, Niels Bohr Institute, Juliane Maries Vej 30, 2100 København Ø, Denmark

17 European Southern Observatory, Alonso de Córdova 3107, Santiago 19, Chile

18 Instituto de Astronomía y Física del Espacio, CONICET/UBA, Casilla de Correos 67, Suc. 28, (1428) Buenos Aires, Argentina

19 JILA, Campus Box 440, University of Colorado, Boulder, CO 80309-0440, USA

Received 7 November 2007 / Accepted 31 October 2008

ABSTRACT

Aims. The aim of this paper is to investigate the environment of gamma ray bursts (GRBs) and the interstellar matter of their host galaxies.

Methods. To this purpose we use high resolution spectroscopic observations of the afterglow of GRB050922C, obtained with UVES/VLT $3.5 \mathrm{~h}$ after the GRB event.

Results. We found that, as for most high resolution spectra of GRBs, the spectrum of the afterglow of GRB050922C is complex. At least seven components contribute to the main absorption system at $z=2.1992$. The detection of lines of neutral elements like MgI and the detection of fine-structure levels of the ions FeII, SiII and CII allows us to separate components in the GRB ISM along the line of sight. Moreover, in addition to the main system, we analyzed the five intervening systems between $z=2.077$ and $z=1.5664$ identified along the GRB line of sight.

Conclusions. GRB afterglow spectra are very complex, but full of information. This can be used to disentangle the contribution of the different parts of the GRB host galaxy and to study their properties. Our metallicity estimates agree with the scenario of GRBs exploding in low metallicity galaxies

Key words. gamma rays: bursts - cosmology: observations - galaxies: abundances - galaxies: ISM

\section{Introduction}

Soon after the discovery of the cosmological origin of gammaray bursts (GRBs) it was realized that they provide powerful tools for investigating the high redshift Universe. Minutes to hours after a GRB, its optical afterglow can sometimes be as bright as magnitude 13-18, such that it can be used as a bright

\footnotetext{
* Based on observations collected at the European Southern Observatory (ESO) with the VLT/Kueyen telescope, Paranal, Chile, in the framework of program 075.A-0603.

$\star \star$ On leave from CEA Saclay, France.
}

beacon to gather high resolution (a few tens of $\mathrm{km} \mathrm{s}^{-1}$ in the optical band), high quality (signal to noise $>10$ per resolution element) spectra. Absorption lines produced by the matter along the line of sight are superimposed on these spectra and allow a detailed investigation of the medium surrounding the GRB and of the host galaxy interstellar matter (ISM).

As of today, about a dozen GRBs have been observed with high resolution spectrographs (and less than one hundred with standard low resolution spectrographs, see Savaglio 2006, and references therein). These high resolution spectroscopic observations showed that the absorption systems along GRB 
sightlines are complex, with many components. They are resolved down to a width of a few tens $\mathrm{km} \mathrm{s}^{-1}$ and contribute to each main absorption system, spanning a total velocity range of typically hundreds $\mathrm{km} \mathrm{s}^{-1}$. In one case, GRB021004, all the systems likely to be associated to the GRB host galaxy span a velocity range of a few thousands $\mathrm{km} \mathrm{s}^{-1}$, although Chen et al. (2007) suggest that the high velocity $3000 \mathrm{~km} \mathrm{~s}^{-1}$ CIV component may no not be related to the GRB host galaxy. Both low and highionization lines are normally observed, as well as fine structure lines of CII, SiII, OI, FeII etc. This is at odds with what is observed from high resolution spectroscopy of QSO "associated" absorption lines (AALs, D'Odorico et al. 2004). First, the equivalent widths and the inferred optical depths along GRB sightlines are much greater than both QSO AALs and those associated with galaxies along QSO sightlines. Second, despite more than 30 years of investigations, only sparse detections of finestructure lines are available along QSOs sightlines due to a lower density environment around them. Fine structure lines have been detected only in Broad Absorption Line QSO spectra (Srianand \& Petitjean 2001, 2000; Hall et al. 2002).

The main reasons for these marked observational differences are probably linked to the characteristics of the phenomena and to their host galaxies. QSOs are long-lasting sources that ionize, partially or totally, the gas over their entire host galaxy. Instead, GRBs are transient phenomena, affecting only smaller regions, close to the site of the GRB explosion. Moreover, the star-forming regions surrounding GRBs are probably much richer in enriched gas than the giant elliptical galaxies, which are the typical host of luminous QSOs (Savaglio 2006). On the other hand, faint metal line systems along QSO sightlines probe mainly galaxy haloes, rather than their bulges or discs. Therefore, GRBs constitute a complementary tool to investigate the ISM of typical high redshift galaxies.

After the first pioneering programs that used BeppoSAX and HETE2 triggers to obtain high resolution spectroscopy of the optical afterglows 12-24 h after the bursts (e.g. Fiore et al. 2005), we are now in a "golden age" for this kind of studies. This is because of the GRB-dedicated Swift satellite which provides accurate GRB positions on time scales as short as tens of seconds, and the rapid response mode (RRM) of the ESO/VLT. Using this new revolutionary observing mode it is possible to obtain GRB afterglow spectra from an $8 \mathrm{~m}$ class telescope with delays from $\sim 10$ min down to a few hours after the GRB event.

The absorption systems along GRB sightlines can be divided into three broad categories: (1) systems associated with the GRB surrounding medium. The physical, dynamical and chemical state of the medium in the star-forming region hosting the GRB progenitor can be modified by the GRB (long type), through shock waves and ionizing photons. Strong fine structure lines are always observed in these systems. Physical and geometrical parameters for the absorbers can be derived comparing the observed line ratios to those predicted by ionization codes (e.g. Nicastro et al. 1999; Perna \& Lazzati 2002; Lazzati et al. 2006; Vreeswijk et al. 2007); (2) Systems associated with random clouds of the ISM of the host galaxy along the line of sight. These systems show low ionization lines and ground state lines, implying distances from the GRB explosion site larger than a few hundred pc; (3) Systems originating in the intergalactic matter along the line of sight.

The distinction between the first and second category is not always clear cut. Dessauges-Zavadsky et al. (2006) analyzed the afterglow spectra of GRB 020813 obtained in two epochs. They report a decline by a factor of 5 in the equivalent width of the Fe II $\lambda 2396$ transition, giving an estimate on its distance from the afterglow of about 50-100 pc. Vreeswijk et al. (2007) discovered large variations of FeII fine-structure lines in the spectra of GRB060418 on rest frame time scales of a fraction of an hour, and interpreted them as due to ultraviolet (UV) pumping from the afterglow radiation field. Their detailed modeling of the observed variability suggests a distance of the FeII absorbing cloud from the GRB explosion site of $\sim 1.7 \mathrm{kpc}$, comparable to the size of a typical galaxy at $z \sim 1.4$. This would imply that, at least in this case, the entire galaxy (or a significant fraction of it) is affected by the GRB explosion. Recently D'Elia et al. (2008) analyzed the high resolution spectra of GRB080319B afterglow 9 min and $8 \mathrm{~h}$ after the GRB onset. They found the strongest Fe II fine structure lines ever observed in a GRB. A few hours later the optical depth of these lines was reduced by a factor of 4-20, and the optical/UV flux by a factor of $\sim 60$. This proves that the excitation of the observed fine structure lines is due to "pumping" by the GRB UV photons. A comparison of the observed ratio between the number of photons absorbed by the excited state and those in the Fe II ground state suggests that the six absorbers are ¿18-34 kpc from the GRB site.

Our goal is to better disentangle different absorption systems by using Ultra-violet and Visual Echelle Spectrograph (UVES, Dekker et al. 2000) observations of GRB050922C. The burst was detected by Swift on 2005 September 22 19:55:50 UT (Norris et al. 2005) and, after 4 s, by Konus/Wind (Golenetskii et al. 2005). ROTSE discovered a bright transient source at $\operatorname{RA}(\mathrm{J} 2000)=21^{\mathrm{h}} 09^{\mathrm{m}} 33^{\mathrm{s}} .1$ and $\operatorname{Dec}(\mathrm{J} 2000)=-08^{\circ} 45^{\prime} 29^{\prime} .8$, $172.4 \mathrm{~s}$ after the burst, which was promptly identified as the optical afterglow of GR050922C (Rykoff et al. 2005) with a magnitude $R=14.7$. The redshift of the GRB, $z=2.1992$, was determined by Jakobsson et al. (2005) using ALFOSC at the Nordic Optical Telescope at the Observatorio del Roque de los Muchachos in La Palma. This redshift was confirmed shortly after through TNG/DOLoRes spectroscopy (Piranomonte et al. 2005). The afterglow was then observed by UVES/VLT at high spectroscopic resolution $(R \sim 40000)$ about $3.5 \mathrm{~h}$ after the burst, when its magnitude was $R=19.0$. The spectrum of GRB050922C is especially rich of features and contains clear examples of the three system categories discussed above. In addition to the main system at $z=2.1992$, there are five intervening systems at $z=1.5664,1.9891,2.0087,2.077,2.1421$ (confirmed also by Sudilovsky et al. 2007).

The system associated with the host galaxy presents several components, some showing strong high-ionization lines and strong fine-structure lines, some showing only low-ionization transitions, thus suggesting that we detect in this GRB both the circum-burst medium and random clouds of the host galaxy ISM along the line of sight.

This paper is organized as follows. Section 2 presents the observations and data reduction; Sect. 3 gives a brief summary of all absorbing systems identified in the UVES spectrum; Sect. 4 presents a detailed analysis of the absorption features belonging to the main absorption system at $z=2.1992$; finally, in Sect. 5 the results are discussed and conclusions are presented.

\section{Observations and data analysis}

\subsection{VLT observations}

In the framework of ESO program 075.A-0603 we observed the afterglow of GRB050922C with UVES mounted at the VLT-UT2 telescope. Table 1 gives the $\log$ of the observations. Both UVES dichroics, as well as the red and the blue arms, were used. This allowed us to achieve a particularly wide 
Table 1. Journal of observations.

\begin{tabular}{lccccccc}
\hline \hline $\begin{array}{l}\text { Date } \\
\text { (UT) }\end{array}$ & Dichroic & \multicolumn{2}{c}{ Central wavelength } & Slit width & Seeing \\
$\left({ }^{\prime \prime}\right)$ & $\begin{array}{c}\text { Exposure } \\
(\text { min })\end{array}$ & $\begin{array}{c}\text { Time since GRB } \\
(\mathrm{h})\end{array}$ \\
\hline $09 / 22 / 0500: 07: 17$ & 1 & $3460 \AA$ & $5800 \AA$ & 1 & $\lessgtr 1$ & 50 & 4.15 \\
$09 / 22 / 0501: 02: 09$ & 2 & $4370 \AA$ & $8600 \AA$ & 1 & $\lesssim 1$ & 50 & 5.07 \\
\hline
\end{tabular}
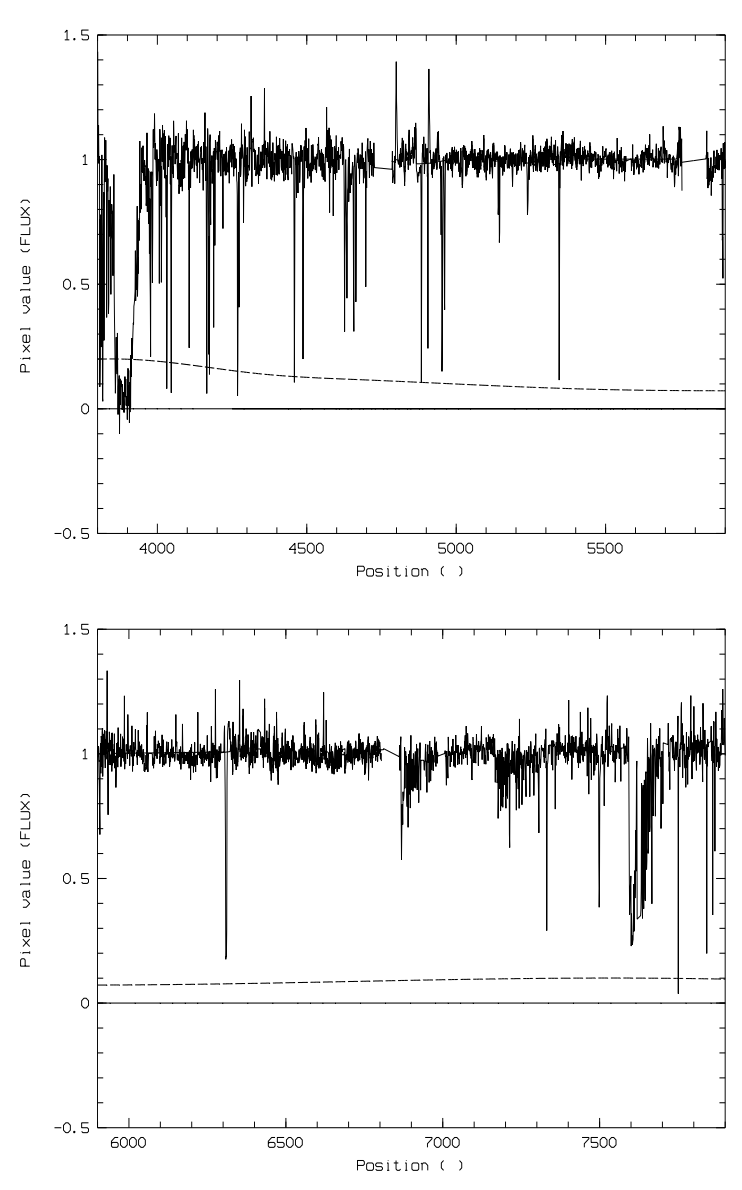

spectral coverage, extending from $\sim 3200 \AA$ to $\sim 10000 \AA$. In order to maximize the signal to noise ratio the $\mathrm{CCD}$ was rebinned in $2 \times 2$ pixels. The data reduction was carried out by using the UVES pipeline (Ballester et al. 2000). The final useful spectra extend from about $3750 \AA$ to about $9780 \AA$. Following Fiore et al. (2005) the total spectrum was rebinned to $0.1 \AA$ to increase the signal-to-noise ratio. This resolution is still good enough to sample with at least 3-4 bins weak lines. The resolution element, set to two pixels, ranges then from $14 \mathrm{~km} \mathrm{~s}^{-1}$ at $4200 \AA$ to $6.6 \mathrm{~km} \mathrm{~s}^{-1}$ at $9000 \AA$. The noise spectrum, used to determine the errors on the best fit line parameters, was calculated from the real-background-subtracted and rebinned spectrum using linefree regions. This takes into account both statistical errors and systematic errors in the pipeline processing and background subtraction.

\subsection{Column densities}

The line fitting was performed using the MIDAS package FITLYMAN (Fontana \& Ballester 1995). This uses a Voigt profile and yields independently the column density $N$ and the Doppler parameter $b$ for each absorption component. For each absorption system several lines, spread over the entire spectral

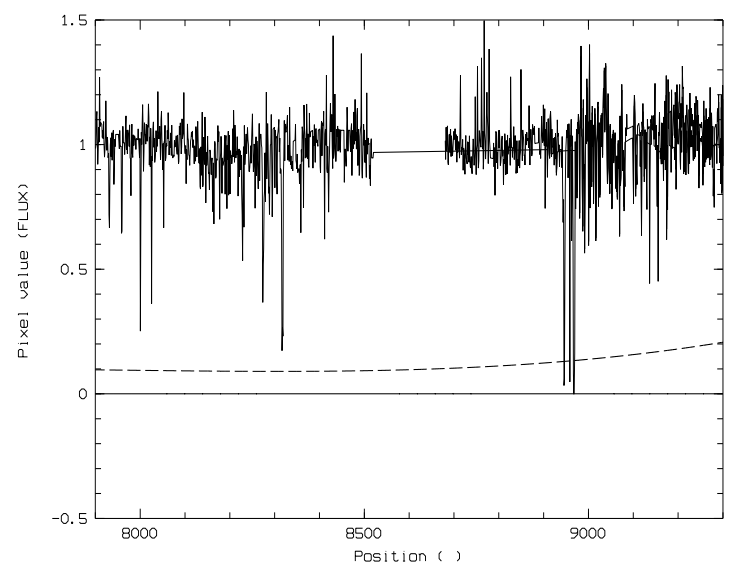

Fig. 1. The full high resolution spectrum of GRB050922C. Dashed lines represent the noise spectrum. range covered by the UVES observations, were fitted simultaneously, using the same number of components for each line, and the same redshift and $b$ value for each component.

In all of the following figures showing plots from FITLYMAN (Figs. 2-10) the $x$-axis represents the velocity shift of the gas with respect to the zero, the $y$-axis represents the flux normalized to 1 for each element, the horizontal blue lines and dashed blue lines represent the flux level of the spectrum normalised to 1 and zero point respectively. The red line corresponds to the fit profile of the individual components and the magenta and green lines correspond to the polynomial approximation of the noise and residual spectrum respectively.

\section{Summary of the absorption systems}

The high resolution spectrum of GRB050922C (see Fig. 1) exhibits a large number of absorption features, belonging to different absorption systems. The main system is due to the gas inferred to be in the host galaxy, at redshift $z=2.1992$. We identified five more intervening absorbers at $z<2.1992(z=1.5664$, $1.5691,1.9891,2.0087,2.077$ and 2.1416). Sudilovsky et al. (2007) found one more intervening systems $z=1.6911$, but we could not confirm that this is real because the C IV doublet is 


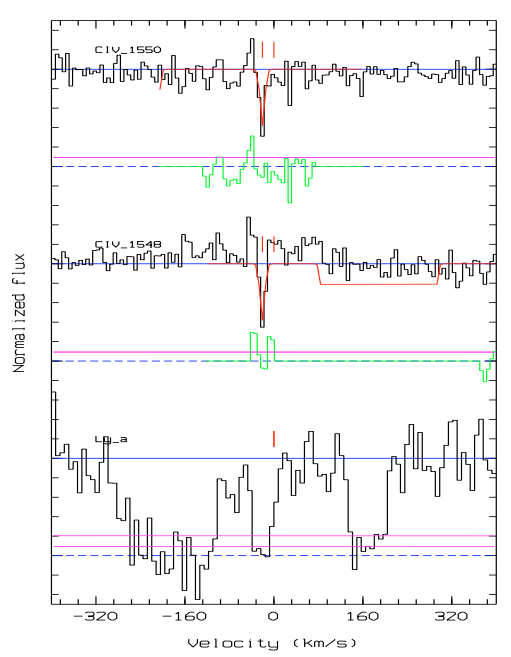

Fig. 2. The intervening absorber at $z=2.1416$. The zero of the velocity scale refers to this redshift.

coincident with the SiII $\lambda 1304$ and OI $\lambda 1302$, OI* $* 1304$ of the main system at $z=2.1992$.

Each system is briefly illustrated in the next subsections.

\subsection{The host galaxy at $z=2.1992$}

This is the system with the highest number of features in the UVES spectrum. Figure 1 shows the Ly- $\alpha$ absorption at $\sim 3870 \AA$ A. The redshift inferred from this feature is confirmed by a high number of metal-line transitions, the narrowest of which allow a more precise determination: $z=2.1992 \pm 0.0005$. This system exhibits a large number of absorption lines, from the neutral hydrogen Ly- $\alpha$ and neutral metal-absorption lines (OI, $\mathrm{NI}$ and FeI) to low-ionization lines (C II, Si II, Mg II, Al II, Fe II, $\mathrm{Al}$ III) and high-ionization absorption features (C IV, Si IV, N v, $\mathrm{PV}, \mathrm{O}$ VI). In addition, strong fine-structure lines (C II*, Si II*, $\mathrm{O} \mathrm{I}^{*}, \mathrm{O}$ II**, ${ }^{*} \mathrm{Fe} \mathrm{II}^{*}, \mathrm{OI} \mathrm{II}^{* *}$ ) have been identified. The total velocity range spanned by this system is $\sim 210 \mathrm{~km} \mathrm{~s}^{-1}$. This system is discussed in detail in the next sections.

\subsection{The intervening absorber at $z=2.1416$}

The intervening system at $z=2.1416$ shows one main component spanning a velocity range of $\sim 20 \mathrm{~km} \mathrm{~s}^{-1}$ (see Fig. 2). This system presents $\mathrm{HI}$ and the CIV transition. The total HI column density is $\log N(\mathrm{HI})=18.1 \pm 0.1 \mathrm{~cm}^{-2}$.

The derived column density of CIV is given in Table 2 .

\subsection{The intervening absorber at $z=2.077$}

The intervening system at $z=2.077$ shows two main components spanning a velocity range of $\sim 50 \mathrm{~km} \mathrm{~s}^{-1}$ (see Fig. 3). This system presents $\mathrm{HI}$ and neutral metal-absorption lines (OI, NI), many low-ionization lines (FeII, SiII, NII, CII) and some high-ionization transitions (SiIII, AlIII, SiIV, CIV). The derived column densities are given in Table 3. We could not separate the two components for HI. The total HI column density is $\log N(\mathrm{HI})=20.3 \pm 0.3 \mathrm{~cm}^{-2}$.
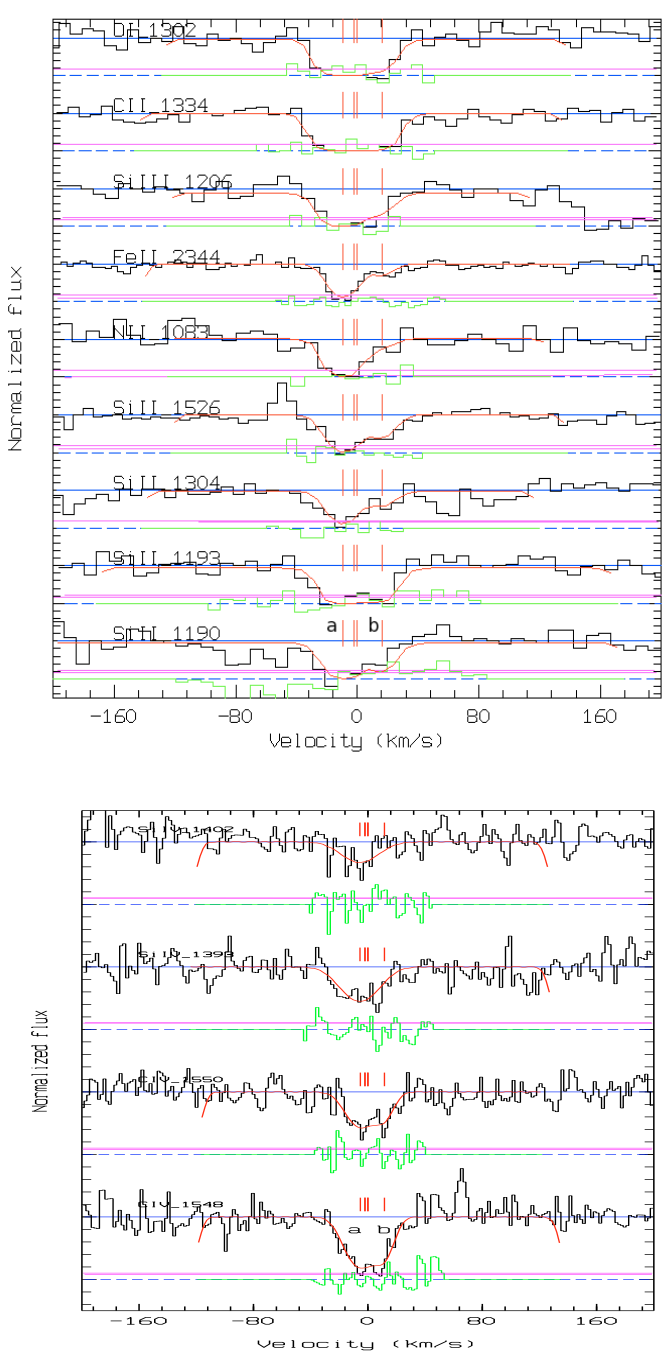

Fig. 3. The intervening absorber at $z=2.077$. The zero of the velocity scale refers to this redshift.

\subsection{The intervening absorber at $z=2.0081$}

The intervening system at $z=2.0081$ shows four main components spanning a velocity range of $\sim 140 \mathrm{~km} \mathrm{~s}^{-1}$ (see Fig. 4). This system presents HI, one low-ionization line (MgII) and some high-ionization transitions (SiIV, CIV). The derived column densities are given in Table 4. We could not separate the four components for HI. The total HI column density is $\log N(\mathrm{HI})=18.2 \pm 0.2 \mathrm{~cm}^{-2}$.

\subsection{The intervening absorber at $z=1.9885$}

The intervening system at $z=1.9885$ shows three main components: $z=1.9885 \mathrm{a}, 1.9885 \mathrm{~b}$ and $1.9885 \mathrm{c}$, spanning a velocity range of $\sim 120 \mathrm{~km} \mathrm{~s}^{-1}$ (see Fig. 5). It presents HI and high-ionization lines (SiIV, CIV). We could not constrain the SiIV 1393 transition because it is blended with the saturated OI 1302 line from the GRB host galaxy. The derived column densities are given in Table 5. We could not separate the three components for HI. The total HI column density is $\log N(\mathrm{HI})=18.2 \pm 0.4 \mathrm{~cm}^{-2}$. 


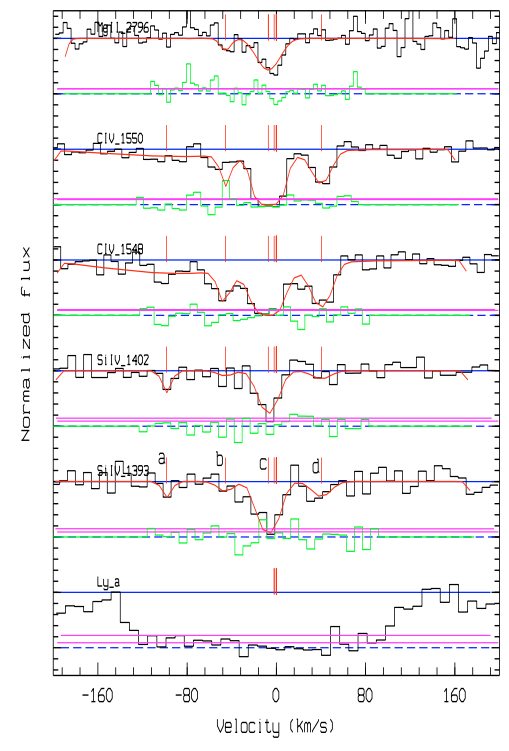

Fig. 4. The intervening absorber at $z=2.0081$. The zero of the velocity scale refers to this redshift.

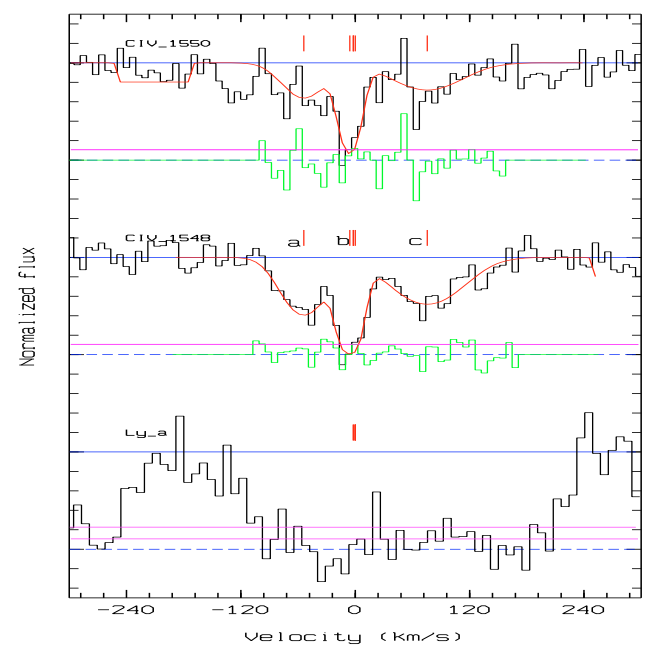

Fig. 5. The intervening absorber at $z=1.9885$. The zero of the velocity scale refers to this redshift.

\subsection{The intervening absorbers at $z=1.5691$ and at $z=1.5664$}

We discuss the intervening system at $z=1.5691$ and $z=1.5664$ together, because the velocity shift between them is very small. They show high ionization line (CIV) and low ionization line (CII), (see Table 6 and Fig. 6).

\section{The $z=2.1992$ absorption system: physical properties of the GRB environment and host galaxy ISM}

This system is most likely associated with the GRB host galaxy. Likewise most GRB host galaxy absorption systems (e.g. Fiore et al. 2005; D'Elia et al. 2007; Vreeswijk et al. 2007; Prochaska et al. 2006, 2008; D'Elia et al. 2008) it presents many components, spanning a rather large velocity range $\left(\sim 210 \mathrm{~km} \mathrm{~s}^{-1}\right)$.

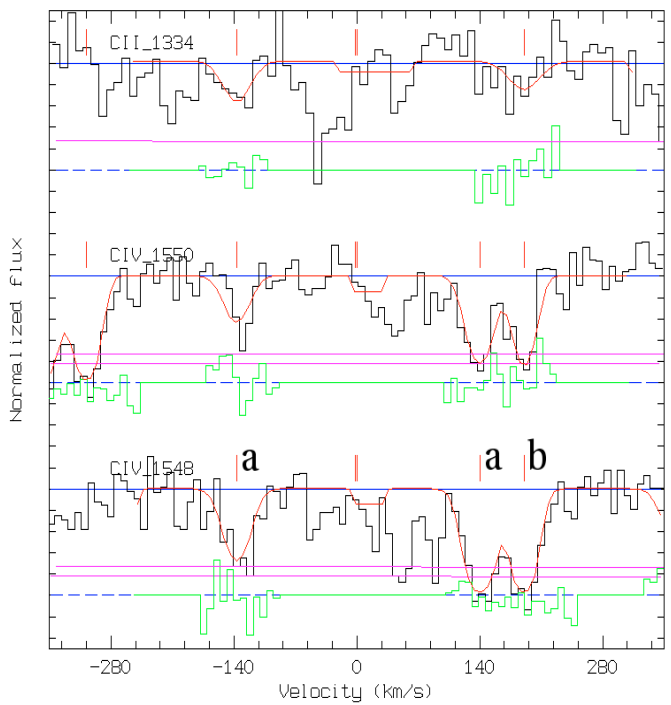

Fig. 6. The intervening absorber at $z=1.5664$ and $z=1.5691$. The zero of the velocity scale refers to a redshift between the two.

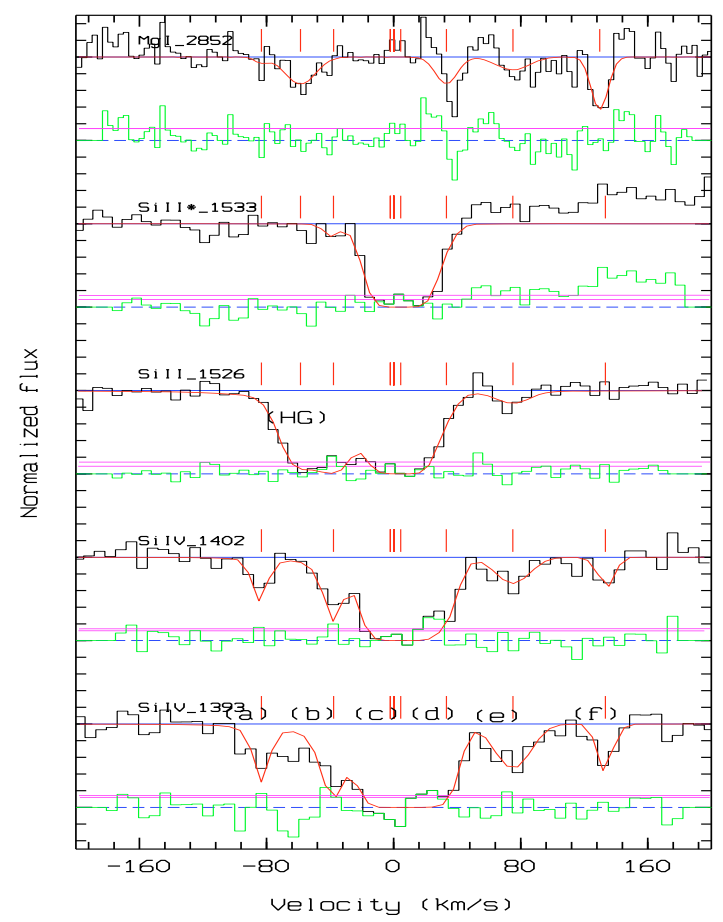

Fig. 7. The UVES spectrum around the lines Si IV $\lambda 1393,1402$, SiII $\lambda 1526$, SiII* $\lambda 1533$ lines and MgL2852. For MgI we clearly see the $(\mathrm{HG})$ component.The zero of the velocity scale refers to the redshift of the host galaxy, $z=2.1992$.

Interestingly, different components exhibit different transitions: some exhibit low-ionization lines, high-ionization lines and finestructure lines, some exhibit low-ionization lines only. An example is given in Fig. 7. Six components, labeled from (a) to (f), are identified for the SiIV and CIV doublets, spanning a velocity range from -75 to $+140 \mathrm{~km} \mathrm{~s}^{-1}$. Each component has a width from 10 to $25 \mathrm{~km} \mathrm{~s}^{-1}$. Component (c) has nearly zero velocity shift and it may be associated with the GRB surrounding medium, because of the strong $\mathrm{Si}$ II* $\lambda 1309$ the highionization transitions and the fact that this component is not 


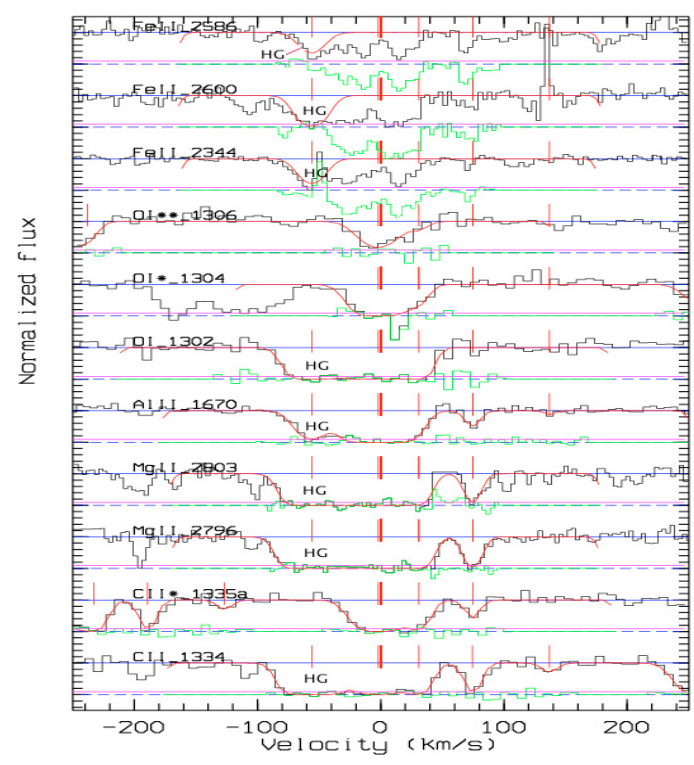

Fig. 8. The "host galaxy" component (HG) is present in all lowionization lines. The column density determination for components a) and b) is difficult due to their blending with the (HG) one.

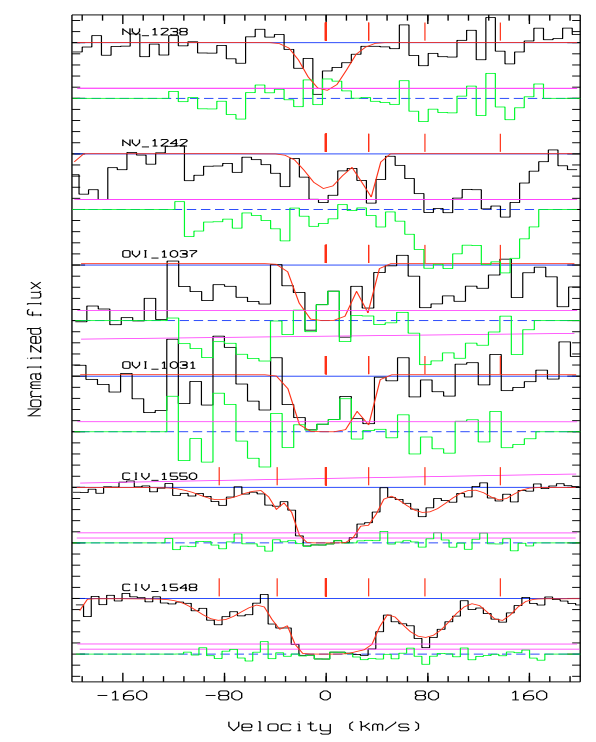

Fig. 9. High-ionization absorption lines for the main system at $z=$ 2.1992. The zero of the velocity scale refers to the redshift of the host galaxy, $z=2.1992$.

detected in MgI. Fainter, but still significant Si II* lines are associated with components (d) and (e) too. On the other hand, the Si II $\lambda 1190$ component labeled (HG) as "host galaxy" at $\sim-58.60 \mathrm{~km} \mathrm{~s}^{-1}$ is not present in either Si IV and Si II* transitions, suggesting that this component is due to some host galaxy cloud along the line of sight not modified by the GRB radiation field. For this component, $\mathrm{Mg} \mathrm{I}$ is seen in absorption (Fig. 7), implying a distance from the GRB site $\gtrsim 100$ pc (Prochaska et al. 2006).

The complex line profile, and the fact that different components may exhibit physically different line transitions, made it impossible to fit the system with a single Voigt profile. Therefore we were forced to identify the different components, before we could derive the column densities through a fitting procedure.

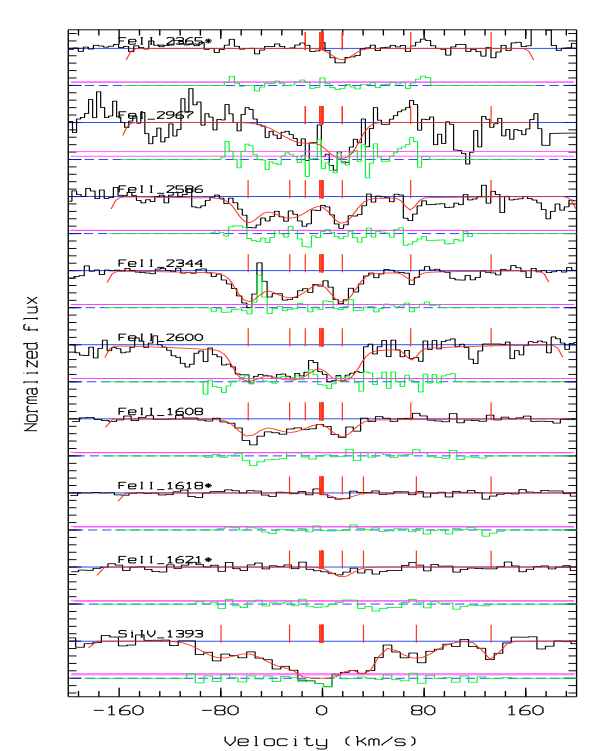

Fig. 10. The UVES spectrum around some FeII and FeII* lines compared with SiIV $\lambda 1393$. The zero of the velocity scale refers to the redshift of the host galaxy, $z=2.1992$.

Table 2. Column densities for the $z=2.1416$ intervening system.

\begin{tabular}{cc}
\hline \hline Transition & 2.1416 \\
\hline CIV & $13.29 \pm 0.07$ \\
\hline
\end{tabular}

Table 3. Column densities for the $z=2.077$ intervening system.

\begin{tabular}{ccc}
\hline \hline Transition & $2.077 \mathrm{a}$ & $2.077 \mathrm{~b}$ \\
\hline FeII & $14.45 \pm 0.08$ & $13.06 \pm 0.04$ \\
SiII & $14.18 \pm 0.08$ & $13.54 \pm 0.07$ \\
NII & $14.56 \pm 0.06$ & $13.26 \pm 0.06$ \\
CII & $15.7 \pm 0.3$ & $14.3 \pm 0.3$ \\
SiII & $13.9 \pm 0.6$ & $12.5 \pm 0.3$ \\
OI & $15.6 \pm 0.3$ & $14.4 \pm 0.2$ \\
NI & $14.2 \pm 0.1$ & $13.4 \pm 0.2$ \\
AlII & - & $12.2 \pm 0.1$ \\
SiIV & $13.05 \pm 0.09$ & $12.5 \pm 0.2$ \\
CIV & $13.70 \pm 0.06$ & $13.3 \pm 0.1$ \\
\hline
\end{tabular}

The main goal of this work is indeed to disentangle the relative contribution of the different components. We use the less saturated transitions to guide this identification. We identified 7 components for the $z=2.1992$ system ((a) through (f), and (HG); see Fig. 7). The redshifts of components (a) to (f), and therefore their velocity shifts with respect to $z=2.1992$, which has been taken as a reference, were determined using the CIV and SiIV lines. The redshift of the (HG) component was determined by using the SiII transitions. The redshifts were taken fixed when fitting all other lines belonging to each component with Voigt profiles. The Doppler parameters of these lines were also linked together. The column densities of non saturated and moderately saturated transitions of each component of the main absorption system at $z=2.1992$ are given in Table 7. Strongly saturated lines could not be used for column density evaluation, and accordingly a "SAT" is given in the tables for the corresponding transitions.

To test the robustness of the results, in terms of the accuracy and stability, we performed many fits, using several combinations of line components. We found that the results presented in 
Table 4. Column densities for the $z=2.0081$ intervening system.

\begin{tabular}{ccccc}
\hline \hline Transition & $2.0081 \mathrm{a}$ & $2.0081 \mathrm{~b}$ & $2.0081 \mathrm{c}$ & $2.0081 \mathrm{~d}$ \\
\hline SiIV & $12.4 \pm 0.2$ & $12.2 \pm 0.3$ & $13.5 \pm 0.1$ & $12.5 \pm 0.2$ \\
CIV & $13.1 \pm 0.1$ & $13.5 \pm 0.1$ & $14.8 \pm 0.1$ & $13.7 \pm 0.1$ \\
MgII & - & $12.0 \pm 0.2$ & $12.64 \pm 0.04$ & - \\
\hline
\end{tabular}

Table 5. Column densities for the $z=1.9885$ intervening system.

\begin{tabular}{cccc}
\hline \hline Transition & $1.9885 \mathrm{a}$ & $1.9885 \mathrm{~b}$ & $1.9885 \mathrm{c}$ \\
\hline CIV & $13.78 \pm 0.05$ & $14.2 \pm 0.1$ & $13.79 \pm 0.04$ \\
\hline
\end{tabular}

Table 6. Column densities for the $z=1.5664$ and 1.5691 intervening systems.

\begin{tabular}{ccc}
\hline \hline Transition & $1.5664 \mathrm{a}$ & \\
\hline CII & $13.6 \pm 0.2$ & \\
CIV & $13.70 \pm 0.07$ & \\
\hline Transition & $1.5691 \mathrm{a}$ & $1.5691 \mathrm{~b}$ \\
\hline CII & - & $13.4 \pm 0.3$ \\
CIV & $14.16 \pm 0.08$ & $14.1 \pm 0.1$ \\
\hline
\end{tabular}

Table 7 provide a good compromise between increasing the statistical precision of the fit, obtained by increasing the number of components fitted simultaneously, and the stability/repeatability of the results. The latter is degraded when the number of fitted parameters is increased, because of the increasingly complex shape of the $\chi^{2}$ hypersurface in the parameter space, which may contain many local minima. We verified that the total best-fit column density of each system is stable, within the statistical errors, changing the number of components in each system.

\subsection{Low-ionization lines}

Strong low-ionization lines were detected for all components. In four cases (MgII, CII, AlII and AlIII) reliable estimates of the ion column density for (c), (d), (e), (f) and (HG) components were possible. In all cases, strong blending of the (a) and (b) components with the $(\mathrm{HG})$ one renders such an estimate difficult for these components. We observe the SiII $(\lambda 1190 \AA, \lambda 1193 \AA$, $\lambda 1260 \AA, \lambda 1304 \AA, \lambda 1526 \AA)$, CII ( $\lambda 1334 \AA)$, MgII doublet $(\lambda 2796 \AA, \lambda 2803 \AA)$, AlII $\lambda 1670 \AA$ and the AlIII doublet ( $\lambda 1854 \AA, \lambda 1862 \AA)$. From Table 7 we can see that the host galaxy component $(\mathrm{HG})$ is present in all low ionization lines (see also Fig. 8).

\subsection{High-ionization lines}

Figure 9 shows all high-ionization lines (NV doublet, OVI doublet) identified together with SiIV and CIV. Significant column densities of CIV and SiIV are detected for all six components (a) to (f). NV and OVI are detected for components (c) and (d) only. Confusion with the Lyman- $\alpha$ forest makes it difficult to search for faint OVI and NV lines for the other components or to compute robust upper limits. No high-ionization lines were detected for component (HG).

\subsubsection{The atoms $\mathrm{Mgl}$ and $\mathrm{Ol}$}

We detect significant amounts of both neutral $\mathrm{Mg}$ and $\mathrm{O}$. The first ionization energy of $\mathrm{Mg}$ lies below the 1 Ryd threshold, at $7.6 \mathrm{eV}$. This transition is not screened by $\mathrm{H}$ absorption and therefore $\mathrm{Mg}$ would be ionized easily by the strong radiation coming from the GRB. As a consequence, the presence of significant $\mathrm{MgI}$ implies large distances ( $>100 \mathrm{pc}$, Prochaska et al. 2006; Chen et al. 2007) of the gas clouds from the GRB site. $\mathrm{MgI}$ is detected for components (HG), (d) and (e) (see Table 7 and Fig. 7). These components are therefore likely to be associated with gas clouds which are little affected by the GRB radiation field. MgI of components (a) and (b) is difficult to constrain because of the blending with the strong $\mathrm{HG}$ component. $\mathrm{MgI}$ is not detected for component (c), which has very high-ionization and fine-structure lines, and for component (f). It should be noted that a line is present at $5.5 \mathrm{~km} \mathrm{~s}^{-1}$ from the position of $\mathrm{MgI}$ at the redshift of component (f), a shift big for this line to be associate to the component.

\subsubsection{Fine-structure lines}

We detected several fine-structure lines associated with components (c), (d), (e) and (f). We identified the following lines arising from fine structure levels: SiII* $21194 \AA$, SiII* $\lambda 1197 \AA$,

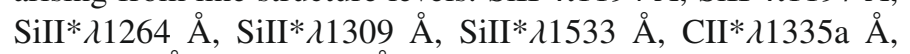
OI* $\lambda 1304 \AA$, OI** $\lambda 1306 \AA$. The redshifts of all these lines were fixed to those of the corresponding components in the CIV and SiIV systems.

We also detect three FeII fine-structure lines: FeII* $\lambda 1618 \AA$, FeII* $\lambda 1621 \AA$, FeII* $\lambda 2365 \AA$. The redshift of these lines is shifted from that found for component (c) using the CIV and $\mathrm{SiV}$ systems by about $20 \mathrm{~km} \mathrm{~s}^{-1}$, see Fig. 10. This suggests that additional structure is actually present in this absorption system. In the following we consider the FeII fine-structure lines as associated with component (c) without making further splitting in velocity. No FeII fine-structure transition is found for the other six components and, in particular, no fine-structure line was found at a redshift consistent with that of component (HG).

\section{Properties of the medium surrounding the GRB}

\subsection{Fine-structure line excitation I: constraining the density of the gas}

As shown in Sect. 4.2.2, for this burst we found several finestructure transitions. These levels can be populated (1) indirectly through excitation by ultraviolet photons, followed by fluorescence, (2) through collisions between the ion and other particles like free electrons, (3) and/or via direct photo-excitation by infrared photons. The first mechanism has been conclusively shown to be the preferred mechanism in two cases: GRB060418 (Vreeswijk et al. 2007) and GRB080319B (D'Elia et al. 2008). The latter mechanism is the less dominant one and we will not consider it. Whatever the case, the physical conditions of the interstellar medium can be probed through the detection of transitions from these energetically lower excited levels.

For GRB050922C fine-structure transitions are present in component (c), (d), (e) and (f), (see Table 7).

If we assume that collisions with electrons are the dominant excitation mechanism, we can put constraints on the electron density of the components where we found such transitions. A method to estimate the electron density is presented by Prochaska et al. (2006). We can compare our results on the SiII*/SiII and FeII*/FeII ratios with Fig. 9 of Prochaska et al. (2006), showing collisional excitation of the first excited state relative at the ground state for $\mathrm{Si}^{+}, \mathrm{Fe}^{+}$and $\mathrm{O}^{0}$. We can only use $\mathrm{Si}$ II and Fe II because oxygen is satured. From this comparison 
Table 7. Derived column densities for the $z=2.1992$ system.

\begin{tabular}{|c|c|c|c|c|c|c|c|}
\hline Transition & $2.1992 \mathrm{a}$ & $2.1992(\mathrm{HG})$ & $2.1992 b$ & $2.1992 \mathrm{c}$ & $2.1992 d$ & $2.1992 \mathrm{e}$ & $2.1992 \mathrm{f}$ \\
\hline $\mathrm{km} \mathrm{s}^{-1}$ & -83.26 & -58.60 & -37.88 & -0.39 & +33.15 & +77.54 & +136.73 \\
\hline MgI & $\dagger$ & $11.8 \pm 0.1$ & $\dagger$ & $<11.8$ & $12.0 \pm 0.1$ & $11.6 \pm 0.2$ & $\star$ \\
\hline OI & - & $15.4 \pm 0.3$ & - & $\ddagger$ & $\ddagger$ & - & - \\
\hline OI* & - & - & - & $15.1 \pm 0.1$ & $14.0 \pm 0.2$ & $<13.7$ & - \\
\hline $\mathrm{OI}^{* *}$ & - & - & - & $14.53 \pm 0.08$ & $13.4 \pm 0.3$ & $<13.7$ & - \\
\hline MgII & $\dagger$ & $14.6 \pm 0.3$ & $\dagger$ & $14.8 \pm 0.6$ & $13.6 \pm 0.3$ & $13.4 \pm 0.3$ & $11.3 \pm 0.5$ \\
\hline CII & $\dagger$ & $15.5 \pm 0.4$ & $\dagger$ & $14.6 \pm 0.2$ & $14.6 \pm 0.5$ & $14.2 \pm 0.3$ & $13.06 \pm 0.21$ \\
\hline CII* & - & $<13.3$ & - & $14.6 \pm 0.1$ & $14.2 \pm 0.3$ & $13.5 \pm 0.2$ & $<13.3$ \\
\hline SiII & $\dagger$ & $14.26 \pm 0.07$ & $\dagger$ & $14.8 \pm 0.1$ & $13.9 \pm 0.2$ & $13.5 \pm 0.2$ & $12.9 \pm 0.2$ \\
\hline SiII* & - & $<13.0$ & - & $14.28 \pm 0.04$ & $13.1 \pm 0.1$ & $13.1 \pm 0.1$ & $12.4 \pm 0.3$ \\
\hline AlII & $\dagger$ & $12.90 \pm 0.05$ & $\dagger$ & $14.0 \pm 0.3$ & $12.1 \pm 0.2$ & $12.15 \pm 0.07$ & $10.6 \pm 0.5$ \\
\hline AlIII & $\dagger$ & $11.8 \pm 0.1$ & $\dagger$ & $12.09 \pm 0.04$ & $11.30 \pm 0.3$ & - & - \\
\hline CIV & $13.40 \pm 0.05$ & $<13.26$ & $13.15 \pm 0.09$ & $15.0 \pm 0.2$ & $13.63 \pm 0.07$ & $13.78 \pm 0.03$ & $13.12 \pm 0.07$ \\
\hline SiIV & $13.07 \pm 0.07$ & $<13.17$ & $13.2 \pm 0.1$ & $14.3 \pm 0.2$ & $13.3 \pm 0.1$ & $13.06 \pm 0.06$ & $12.76 \pm 0.09$ \\
\hline NV & - & - & - & $14.06 \pm 0.08$ & $15.3 \pm 0.7$ & - & - \\
\hline OVI & - & - & - & $15.2 \pm 1.9$ & $14.6 \pm 0.2$ & - & - \\
\hline $\mathrm{Fe}$ & $2.1992 \mathrm{a}$ & 2.1992(HG) & $2.1992 b$ & $2.1992 \mathrm{c}(1)-2.1992 \mathrm{c}(2)$ & $2.1992 \mathrm{~d}$ & $2.1992 \mathrm{e}$ & $2.1992 \mathrm{f}$ \\
\hline $\mathrm{km} \mathrm{s}^{-1}$ & -83.26 & -58.60 & -37.88 & $-13.3 /+15.74$ & +33.15 & +77.54 & +136.73 \\
\hline FeII & - & $13.67 \pm 0.04$ & $13.75 \pm 0.03$ & $13.3 \pm 0.1 / 13.73 \pm 0.04$ & - & $14.3 \pm 0.4$ & - \\
\hline FeII* & - & - & - & $-/ 13.36 \pm 0.07$ & - & - & - \\
\hline
\end{tabular}

$\dagger$ This component is blended with the host galaxy component (HG), so we cannot determine the relative column density. $\$$ Saturated component.

$\star$ Component confused by another one shifted to $5.5 \mathrm{~km} \mathrm{~s}^{-1}$ with respect to (f) position.

we find for SiII $n_{\mathrm{e}}$ in the range $(1.3-3) \times 10^{2} \mathrm{~cm}^{-3}$ for component $(\mathrm{c}), n_{\mathrm{e}}$ in the range $(1.2-2) \times 10^{2} \mathrm{~cm}^{-3}$ for component $(\mathrm{d})$, $n_{\mathrm{e}}<2 \times 10^{1} \mathrm{~cm}^{-3}$ for component (e) and $n_{\mathrm{e}}>2 \times 10^{3} \mathrm{~cm}^{-3}$ for component (f).

We have evaluated the electron density of component (c) also using the Fe II transitions. This produces a result $n_{\mathrm{e}} \sim$ $(0.1-5) \times 10^{5} \mathrm{~cm}^{-3}$ inconsistent with the previous estimate. A possible reason for this inconsistency may be our simple description of a complex system (the excited transition lines are shifted by $20 \mathrm{~km} \mathrm{~s}^{-1}$ from the ground level lines).

\subsection{Fine-structure line excitation II: constraining the radiation field}

A competitive mechanism responsible for the presence of finestructure levels is indirect UV pumping. If this is the dominant excitation mechanism, we can constrain the GRB radiation field and the distance of the gas from the GRB. Prochaska et al. (2006) using the PopRatio code developed by Silva \& Viegas (2001, 2002), analyzed the relation between the far UV radiation field intensity and the relative fraction of excited fine-structure states with respect to their ground levels (for O I, Si II and Fe II, see their Figs. 7 and 8). In the following we make use of these figures to estimate the radiation-field intensity from our column density data.

Using Fig. 7 of Prochaska et al. (2006), we find that our measured ratio $\log \left(\mathrm{Si} \mathrm{II}^{*} / \mathrm{SiII}\right)=-0.5 \pm 0.1$ for component (c) implies a radiation field intensity $G / G_{0}=2_{-1}^{+3} \times 10^{5}$ ( $90 \%$ confidence interval; $G_{0}=1.6 \times 10^{-3} \mathrm{erg} \mathrm{cm}^{-2} \mathrm{~s}^{-1}$ is the Habing constant). We note that the Si II lines of this component are moderately saturated and therefore that additional systematic uncertainty may affect their column densities determination. Fortunately, for this component we detect also Fe II and Fe II* transitions. The $\log \left(\mathrm{Fe} \mathrm{II}^{*} / \mathrm{Fe} \mathrm{II}\right)$ is $-0.5 \pm 0.1$, implying a radiation field intensity $G / G_{0}=6_{-5}^{+4} \times 10^{6}$, consistent with the Si II determination, within the rather large statistical errors.

A similarly high value of $G / G_{0}$ is found for component (f) only, for which we found $\log \left(\mathrm{Si} \mathrm{II}^{*} / \mathrm{Si} \mathrm{II}\right)=-0.5 \pm 0.3$, implying a radiation field intensity of $G / G_{0}=3_{-0.4}^{+5} \times 10^{5}$.

For component (d) and (e) we found $\log \left(\mathrm{Si} \mathrm{II}^{*} / \mathrm{Si} \mathrm{II}\right)=$ $-0.8 \pm 0.2$ and $-1.4 \pm 0.4$ respectively, implying radiation field intensity $G / G_{0}=(8 \pm 1) \times 10^{4}$ and $G / G_{0}=1_{-0.7}^{+0.7} \times 10^{4}$, both significantly lower than for component (c). We note that the Si II lines of component (e) are certainly not saturated or blended with other components, while that of component (d) may be slightly blended and/or saturated.

While UV pumping is the likely mechanism for the population of fine structure levels in this GRB spectrum, we cannot rule out collisional exitation. The "smoking gun" of the UV pumping mechanism is time variability of fine structure lines (Vreeswijk et al. 2007; D'Elia et al. 2008). Unfortunately we have only one single observation of the GRB050922C and therefore we cannot look for line variations. We note that components (c) and (f) experience the highest radiation field.

\section{Discussion}

\subsection{Distances from the burst region}

We plot in Fig. 11 high to low-ionization line ratios and fine structure to ground state line ratios for the seven components of the $z=2.1992$ system. The different behavior of component (HG) with respect to all the others and in particular to components (c) and (f) is evident from this plot. The most natural explanation for this behavior is to assume that the various components do not belong to the same physical region in the host galaxy. The strength of the high ionization lines and of the finestructure lines, the absence of MgI absorption, the analysis of the ratio of the excited levels to ground levels and the metallicity analysis suggest that components (c) and (f) are closer 


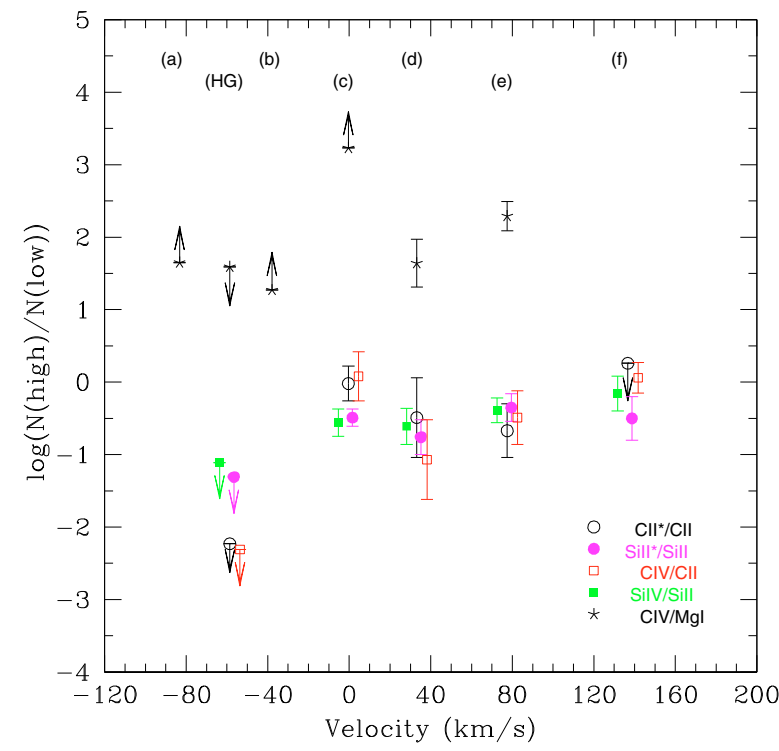

Fig. 11. Several high- to low-ionization ion column densities ratios for the seven components of the $z=2.1992$ system as a function of the velocity shift with respect to the redshift of the host galaxy. See text for details.

to the GRB site than the other components. To better quantify this suggestion we performed detailed time evolving photoionization calculations using the GRB light-curve to estimate the photoionization fractions (assuming an optically thin medium). We used the code initially developed by Nicastro et al. (1999) and updated by Krongold et al. (2008). We assumed a constant density profile throughout the cloud, and a plane parallel geometry. We studied gas densities between $10 \mathrm{~cm}^{-3}$ and $10^{8} \mathrm{~cm}^{-3}$. The ionizing continuum was assumed to be a power law, $F(E)=E^{-\Gamma}$ photons $\mathrm{cm}^{-2} \mathrm{~s}^{-1}$, with cutoffs at low and high energy. The high energy cutoff was fixed at $10^{21} \mathrm{~Hz}$.

Our calculations require that component (c), in order to reproduce simultaneously the lines by OVI, NV, CIV, SiIV, FeII and OI, the gas density would have to be between $10^{5}$ and $10^{7} \mathrm{~cm}^{-3}$, and the distance between 200 and $300 \mathrm{pc}$ from the GRB site ${ }^{1}$

The presence of neutral elements in the spectra of GRB afterglows can place strong constraints on the distance of the gas from the GRB. For example, Prochaska et al. (2006) suggest that components showing MgI absorption should be located at distances greater than $100 \mathrm{pc}$ from the GRB site. In the afterglow spectra of GRB050922C we detect neutral MgI for three components: (HG), (d) and (e), see Fig. 7. In particular, the presence of strong $\mathrm{MgI}$ absorption, absence of high-ionization lines and excited transitions, and low metallicity, suggest that the (HG) component is located further away from the GRB site, in a region of the host galaxy not strongly affected by the GRB radiation field. Our time evolving photoionization calculations require a distance of at least $700 \mathrm{pc}$ from the GRB site for this component. Components (d) and (e) do show fine-structure transitions. If the dominant excitation mechanism is UV pumping from the GRB radiation field, this suggests a distance $\lesssim 1 \mathrm{kpc}$ from the GRB site (Prochaska et al. 2007). Our time evolving photoionization calculations require a distances $\gtrsim 300 \mathrm{pc}$ for both components, to be consistent with the observed $\mathrm{C}$ and $\mathrm{Si}$ line ratios. Component (d) exhibits also high-ionization absorption (NV, PV, OVI). The

\footnotetext{
1 This density is implied only if both the high and low ionization lines come from the same gas phase.
}

Table 8. Metallicity.

\begin{tabular}{|c|c|c|}
\hline Transition & $\overline{l o g} N^{a}\left(\mathrm{~cm}^{-2}\right)$ & 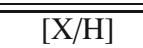 \\
\hline HI & $21.3 \pm 0.4$ & - \\
\hline $\mathrm{C}$ & $15.8 \pm 0.5$ & $-1.9 \pm 0.5$ \\
\hline $\mathbf{S i}$ & $15.3 \pm 0.5$ & $-1.5 \pm 0.5$ \\
\hline $\mathbf{O}$ & $15.7 \pm 0.3$ & $-2.3 \pm 0.3$ \\
\hline $\mathbf{N}$ & $14.9 \pm 0.7$ & $-2.3 \pm 0.7$ \\
\hline Al & $14.2 \pm 0.5$ & $-1.5 \pm 0.5$ \\
\hline Mg & $14.9 \pm 0.3$ & $-1.9 \pm 0.3$ \\
\hline $\mathrm{Fe}$ & $14.9 \pm 0.4$ & $-2.1 \pm 0.4$ \\
\hline
\end{tabular}

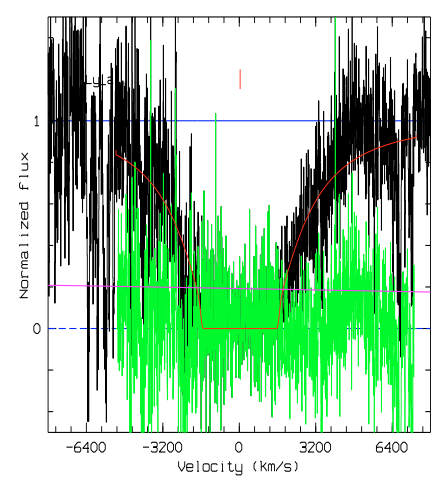

Fig. 12. The Ly- $\alpha$ absorption feature.

presence of these highly ionized ions, along with the presence of low ionized ones (O I, Mg I, Fe II), can be explained at a distance of $\sim 300 \mathrm{pc}$ from the GRB site if the density of this cloud is high enough, i.e. $>10^{5} \mathrm{~cm}^{-3}$.

For components (a) and (b) we do not have information on the abundance of neutral elements, because these transitions occur at wavelenghts affected by the strong absorption of component (HG). The detection of CIV implies a distance to the GRB site closer than $\sim 700 \mathrm{pc}$ for both components (a) and (b).

\subsection{Carbon and iron abundances}

Following D'Elia et al. (2007), we calculated the average $[\mathrm{C} / \mathrm{Fe}]$ ratio, an estimator of the enrichment of the $\alpha$ elements relative to iron. We measure a mean $[\mathrm{C} / \mathrm{Fe}]=0.38 \pm 0.75$, consistent with the value predicted by the models of Pipino \& Matteucci $(2004,2006)$ for a galaxy younger than 1 Gyr subject to a burst of star-formation. In such a case also a low $[\mathrm{Fe} / \mathrm{H}]$ value is predicted, close to that in Table 8 , and similar to what found by D'Elia et al. (2007) for GRB050730. Again, we note that mean abundance estimates may be affected by large systematic uncertainties.

\subsection{Metallicity of the main system}

Figure 12 shows the Ly- $\alpha$ absorption features. Hydrogen absorption results in very broad lines with damped wings, reaching well beyond the velocity range of the heavier ions. Therefore is clearly impossible to separate the seven components identified from the analysis of C IV, Si IV and Si II, and we can only estimate the metallicity of the host galaxy in terms of the ratio $[\mathrm{X} / \mathrm{H}]$ between the total absorption column of the $\mathrm{X}$ element with respect to that of hydrogen. 
For this burst we measure a Hydrogen column density $\log N_{\mathrm{HI}}=21.3 \pm 0.4 \mathrm{~cm}^{-2}$, similar to the average value found by Jakobsson et al. (2006) for a sample of Swift GRBs.

Table 8 gives, for each element, the total column density of the seven components obtained by adding the column densities for each ion (including ground states and the excited finestructure levels, if these are present) and the metallicity of the element on a log scale. Taken at face value these metallicities are rather small. Furthermore, a fraction of Hydrogen may well be ionized, in particular that associated to the components closer to the GRB site (Waxman \& Draine 2000; Prochaska et al. 2007), suggesting even lower metallicities. On the other side, the quoted metallicities are not corrected for dust depletion. This is likely to be particuarly important for iron (see Savaglio 2006) while it should be negligible for Silicon. The Si metallicity in Table 8 is formally higher than that of $\mathrm{Fe}$, but statistically consistent within the large error bars. In conclusion, even considering a little contribution from ionized Hydrogen, we can exclude metallicities higher than $\approx 1 / 10$ solar.

This GRB was observed by the Swift X-ray telescope (XRT) Swift at the same time of the UVES observations. It is therefore possible to compare directly the column density determined from the Ly- $\alpha$ observation to that obtained from X-ray spectroscopy. The spectrum was fitted using model including a power law reduced at low energy by intervening absorption at the redshift of the GRB host galaxy. An additional local absorber was included in the fit to account for Galactic gas along the GRB line of sight. The column density of this absorber was fixed at $5.75 \times 10^{20} \mathrm{~cm}^{-2}$ (Dickey \& Lockman 1990). Solar abundances were adopted for the Galactic absorber while the metallicity of the GRB rest frame column density was fixed either to solar metallicities or to $1 / 10$ solar. The best fit rest frame $N_{\mathrm{H}}$ values are $(4.7 \pm 1.9) \times 10^{21} \mathrm{~cm}^{-2}$, and $(3.0 \pm 1.0) \times 10^{22} \mathrm{~cm}^{-2}$ for the two cases respectively. The latter value is higher than what found using the Lyman- $\alpha$ in the UVES spectrum a result consistent with what found by Jakobsson et al. (2006) from other GRBs.

The metallicity estimates can be compared to metallicity derivations that use optical emission lines from the GRB host galaxy. Savaglio (2006) found an average value of -0.15 for a sample of 11 GRB hosts at $z<1$, a value significantly higher than those in Table 8 . The values in Table 8 are significantly lower than this feature. As discussed above several effects can play a role, the main three being the exact correction for dust depletion, the amount of ionized Hydrogen and the fact that we cannot estimate the $\mathrm{H}$ column density of each single component. This is particularly relevant, since different components can be associated with regions of the host galaxy with very different metallicities. On the other hand, emission line spectroscopy of GRB host galaxies is very difficult to perform at $z>1$, meaning that at high redshift absorption line spectroscopy of GRBs is probably the only tool to estimate host metallicities.

\subsection{Metallicity of the intervening systems}

The Ly- $\alpha$ of three of the five intervening systems lies in the UVES observed band. In these cases we were able to estimate the HI column density (see Sects. 3.2, 3.4 and 3.5), and therefore the metallicity following the same approach used for the main system.

For the $z=2.1416$ intervening system we measure $[\mathrm{C} / \mathrm{H}]=-1.2 \pm 0.1$. For the $z=2.077$ intervening system we measure $[\mathrm{C} / \mathrm{H}]=-1.4,[\mathrm{Si} / \mathrm{H}]=-1.4,[\mathrm{Fe} / \mathrm{H}]=-1.3$, $[\mathrm{N} / \mathrm{H}]=-1.5,[\mathrm{O} / \mathrm{H}]=-1.4$. For this system we can also estimate the SiIV/CIV ratio, which is $-0.7 \pm 0.1$ for component (a) and $-0.8 \pm 0.2$ for component (b). These values are near the median value performed by Songaila (1998). For the $z=2.0081$ intervening system we measure $[\mathrm{C} / \mathrm{H}]=0.7 \pm 0.5,[\mathrm{Si} / \mathrm{H}]=-0.1 \pm 0.4$ and $[\mathrm{Mg} / \mathrm{H}]=-1.0 \pm 0.2$. The SiIV/CIV ratio, which is $-1.30 \pm$ 0.15 for component (c), is similar to the median value computed by Songaila (1998) but at the lower end of the range found by D'Odorico \& Petitjean (2001) for systems at $z \sim 1.9$. Finally, for the $z=1.9885$ intervening system we measure $[\mathrm{C} / \mathrm{H}]=-0.1 \pm 0.5$. These values are not corrected for the fractions of $\mathrm{Si}$ and $\mathrm{C}$ in ions different from those in observed transitions and reported in Tables 2, 4 and 5, and, most important are not corrected for the fraction of $\mathrm{H}$ which may be ionized. This so called "ionization correction" is a rather steep function of the observed HI column density (Péroux et al. 2007), and for low observed column densities, such those for the three intervening systems under study, may be as large as $-0.3--0.4$. Accounting for this ionization correction, the $[\mathrm{C} / \mathrm{H}]$ of the $z=2.0081$ and $z=1.9885$ systems appear significantly higher than that found for the GRB host galaxy in Table 8. They are also higher than the typical metallicity found in DLA and sub-DLA at similar redshifts (Péroux et al. 2007). We remark however that our determinations are based on rather sparse line detections and therefore that a more systematic work is needed to investigate further this issue.

\section{Conclusions}

Using the VLT high resolution spectrometer UVES, we have obtained a high signal to noise $(\sim 20)$ spectrum of the optical afterglow of GRB050922C. Five intervening systems between $z=2.077$ and $z=1.5664$ have been identified along the GRB line of sight, in addition to the main system at $z=2.1992$, which we identify as the redshift of the GRB host galaxy. The spectrum shows that the ISM of this galaxy is complex, with at least seven components contributing to the main absorption system. These systems show both high- and low-ionization lines, fine-structure lines and neutral element absorption lines, thus suggesting wide ranges of distances to the GRB site and physical properties of the absorbing clouds. The analysis of ratios between high and low ionization lines, the presence of strong fine structure line and the analysis of the metallicity of the clouds allow us to put quantitative constraints on the distance of the absorbing clouds to the GRB site. In particular, we find that component (c) is likely to be the closest component, with a distance 200-300 pc from the GRB site. Component HG is likely to be farthest component, with a distance $>700 \mathrm{pc}$.

We calculated the average metallicity of the GRB host galaxy by adding the column densities of all seven components. This turned out to be between $1 / 100$ and $1 / 10$ solar, even for an element like Silicon, which should not be strongly affected by dust depletion. This value is roughly consistent with what is found by absorption spectroscopy in other $z=2-4$ GRBs (Savaglio 2006; Prochaska et al. 2007) but is lower than the average found by emission line spectroscopy at $z<1$ (Savaglio 2006; Savaglio et al. 2008). Interestingly, the $C$ fraction of three intervening systems at $z \sim 2$ is nearly solar, and therefore significantly higher than that of the GRB host galaxy.

The element transitions detected, the complexity of the components and the column densities measured are similar to the other high redshift GRB high resolution spectra present in the literature. We have no evidence of both the high velocity components reported for GRB021004 (Mirabal et al. 2003; Fiore et al. 2005; Starling et al. 2005), GRB030226 (Klose et al. 2004) and 
GRB050505 (Berger et al. 2006), and the strong asymmetry reported for GRB030329 (Thöne et al. 2007).

Acknowledgements. We thank an anonymous referee for comments that improved the presentation. We thank C. Porciani, V. D'Odorico, H.-W. Chen, J. X. Prochaska and P. M. Vreeswijk for useful discussions. Part of this work was supported by MIUR COFIN-03-02-23 and INAF/PRIN 270/2003 and ASI contracts ASI/I/R/039/04 and ASI/I/R/023/05/0. S.P. and V.D. acknowledge support from ASI grants. S.P., S.D.V. and M.D.V. thank the Dark Cosmology Centre of Copenhagen, where part of this work was done, for the friendly and creative atmosphere. D.M. acknowledges the Instrument Center for Danish Astrophysics for support and thanks the Dark Cosmology Centre (funded by the DNRF). S.D.V. is supported by SFI.

\section{References}

Ballester, P., Modigliani, A., Boutquin, O., et al. 2000, Messenger, 101, 31

Berger, E., Penprase, B. E., Cenko, S. B., et al. 2006, ApJ, 642, 979

Chen, H.-W., Prochaska, J. X., \& Bloom, J. S. 2007, ApJ, 668, 384

Dekker, H., D’Odorico, S., Kaufer, A., Delabre, B., \& Kotzlowski, H. 2000, in Optical and IR Telescope Instrumentation and Detectors, ed. M. Iye, \& A. F. Moorwood, Proc. SPIE, 4008, 534

D'Elia, V., Fiore, F., Meurs, E. J. A., et al. 2007, A\&A, 467, 629

D'Elia, V., Fiore, F., Perna, R., et al. 2008, ArXiv e-prints, 804

Dessauges-Zavadsky, M., Chen, H.-W., Prochaska, J. X., Bloom, J. S., \& Barth, A. J. 2006, ApJ, 648, L89

Dickey, J. M., \& Lockman, F. J. 1990, ARA\&A, 28, 215

D'Odorico, V., \& Petitjean, P. 2001, A\&A, 370, 729

D’Odorico, V., Cristiani, S., Romano, D., Granato, G., \& Danese, L. 2004, MNRAS, 424, 23

Fiore, F., D'Elia, V., Lazzati, D., et al. 2005, ApJ, 624, 853

Fontana, A., \& Ballester, P. 1995, ESO Messenger, 80, 37

Golenetskii, S., Aptekar, R., Mazets, E., et al. 2005, GRB Coordinates Network, 4030, 1

Hall, P. B., Gunn, J. E., Knapp, G. R., et al. 2002, in Mass Outflow in Active Galactic Nuclei: New Perspectives, ed. D. M. Crenshaw, S. B. Kraemer, \& I. M. George, ASP Conf. Ser., 255, 161
Jakobsson, P., Fynbo, J. P. U., Paraficz, D., et al. 2005, GRB Coordinates Network, 4017, 1

Jakobsson, P., Fynbo, J. P. U., Ledoux, C., et al, 2006, A\&A, 460, L13

Klose, S., Greiner, J., Rau, A., et al. 2004, AJ, 128, 1942

Krongold, Y., Nicastro, F., Elvis, M., et al. 2008, in Revista Mexicana de Astronomia y Astrofisica Conference Series, 32, 123

Lazzati, D., Perna, R., Flasher, J., Dwarkadas, V. V., \& Fiore, F. 2006, MNRAS, 372, 1791

Mirabal, N., Halpern, J. P., Chornock, R., et al. 2003, ApJ, 595, 935

Nicastro, F., Fiore, F., Perola, G. C., \& Elvis, M. 1999, ApJ, 512, 184

Norris, J., Barbier, L., Burrows, D., et al. 2005, GRB Coordinates Network, 4013, 1

Perna, R., \& Lazzati, D. 2002, ApJ, 580, 261

Péroux, C., Dessauges-Zavadsky, M., D’Odorico, S., Kim, T.-S., \& McMahon, R. G. 2007, MNRAS, 382, 177

Pipino, A., \& Matteucci, F. 2004, MNRAS, 347, 968

Pipino, A., \& Matteucci, F. 2006, MNRAS, 365, 1114

Piranomonte, S., Magazzu, A., Mainella, G., et al. 2005, GRB Coordinates Network, 4032, 1

Prochaska, J. X., Chen, H.-W., \& Bloom, J. S. 2006, ApJ, 648, 95

Prochaska, J. X., Chen, H.-W., Dessauges-Zavadsky, M., \& Bloom, J. S. 2007, ApJ, 666, 267

Prochaska, J. X., Dessauges-Zavadsky, M., Ramirez-Ruiz, E., \& Chen, H.-W. 2008, ArXiv e-prints, 806

Rykoff, E. S., Yost, S. A., Rujopakarn, W., et al. 2005, GRB Coordinates Network, 4012, 1

Savaglio, S. 2006, New J. Phys., 8, 195

Savaglio, S., Glazebrook, K., \& Le Borgne, D. 2008, ArXiv e-prints, 803

Silva, A. I., \& Viegas, S. M. 2001, Comp. Phys. Commun., 136, 319

Silva, A. I., \& Viegas, S. M. 2002, MNRAS, 329, 135

Songaila, A. 1998, AJ, 115, 2184

Srianand, R., \& Petitjean, P. 2000, A\&A, 357, 414

Srianand, R., \& Petitjean, P. 2001, A\&A, 373, 816

Starling, R. L. C., Wijers, R. A. M. J., Hughes, M. A., et al. 2005, MNRAS, 360, 305

Sudilovsky, V., Savaglio, S., Vreeswijk, P., et al. 2007, ArXiv e-prints, 705

Thöne, C. C., Greiner, J., Savaglio, S., \& Jehin, E. 2007, ApJ, 671, 628

Vreeswijk, P. M., Ledoux, C., Smette, A., et al. 2007, A\&A, 468, 83

Waxman, E., \& Draine, B. T. 2000, ApJ, 537, 796 